\title{
Crescimento Pós-Traumático em Profissionais de Emergências: Uma Revisão Sistemática de Estudos Observacionais
}

\author{
Eduardo de Paula Lima ${ }^{1}$ \\ Alina Gomide Vasconcelos ${ }^{1}$ \\ Elizabeth do Nascimento ${ }^{1}$ \\ ${ }^{1}$ Universidade Federal de Minas Gerais, Belo Horizonte, Minas Gerais, Brasil
}

\begin{abstract}
Resumo
A exposição a eventos traumáticos pode gerar Crescimento Pós-Traumático (CPT). O objetivo do presente estudo foi realizar uma revisão sistemática das publicações sobre prevalência de CPT e sua associação com estressores ocupacionais entre profissionais de emergências. A revisão foi baseada no método PRISMA e previamente registrada no PROSPERO. A busca foi realizada nas bases de dados Medical Literature Analysis and Retrieval System Online (Medline), Literatura Latino-Americana e do Caribe em Ciências da Saúde (Lilacs) e Scientific Electronic Library Online (ScIELO). Dentre os resultados, não foi possível identificar a prevalência de CPT. Estressores operacionais foram associados ao CPT. Estressores organizacionais foram raramente investigados. Concluiu-se que o CPT é um dos focos em estudos sobre reações pós-traumáticas entre profissionais de emergências e está associado a estressores ocupacionais. Por isso, trata-se de um construto importante para compreender a saúde mental desses profissionais, dada a organização e a natureza de suas tarefas no trabalho.
\end{abstract}

Palavras-chave: revisão, trauma psicológico, serviços médicos de emergência, bombeiros, polícia

\section{Posttraumatic Growth Among Emergency Workers: A Systematic Review of Observational Studies}

\begin{abstract}
Exposure to traumatic events can lead to Posttraumatic Growth (PTG). The present study aimed to was to conduct a systematic review of publications on the prevalence of PTG and its association with occupational stressors among emergency professionals. The review was based on the PRISMA method and previously registered in PROSPERO. The search was conducted in following eletronic databases: Medical Literature Analysis and Retrieval System Online (Mediline), Latin American and Caribbean Literature in Health Sciences (Lilacs), and Scientific Electronic Library Online (Scielo). It was not possible to identify the prevalence of The prevalence of PTG was not identified among emergency professionals. Operational stressors were associated with to PTG. Organizational stressors were rarely investigated. We concluded that PTG is one of the focuses in studies on post-traumatic reactions among emergency professionals and it is associated with occupational stressors. Therefore, it is an important construct to understand the mental health of these professionals given the organization and the nature of their occupational tasks.

Keywords: review; psychological trauma; emergency medical services; firefighters; police
\end{abstract}

\section{Crecimiento Post-Traumático en Profesionales de Servicios de Urgencia: Una Revisión Sistemática de Estudios Observacionales}

\begin{abstract}
Resumen
La exposición a eventos traumáticos puede generar Crecimiento Post-Traumático (CPT). El objetivo del presente estudio fue realizar una revisión sistemática de las publicaciones sobre predominio del CPT y su asociación con estresores ocupacionales entre profesionales de servicios de urgencia. La revisión fue basada en el método PRISMA y previamente registrada en el PROSPERO. La búsqueda se llevó a cabo en las bases de datos de Medical Literature Analysis and Retrieval System Online (Medline), Literatura Latino-Americana e do Caribe em Ciências da Saúde (Lilacs) e Scientific Electronic Library Online (Scielo). Entre los resultados, no fue posible identificar el predominio del CPT. Estresores operativos se asociaron con el CPT y estresores organizacionales fueron raramente investigados. Se concluyó que el CPT es el foco principal en los estudios sobre reacciones postraumáticas entre los profesionales de servicios de urgencia y está asociado a estresores ocupacionales. Por eso, se trata de un constructo importante para comprender la salud mental de esos profesionales dada la organización y la naturaleza de sus tareas en el trabajo.

Palabras clave: revisión; trauma psicológico; servicios médicos de urgencia; bomberos; policía
\end{abstract}

\section{Introdução}

Guerras, desastres e catástrofes naturais têm efeitos nocivos sobre o funcionamento psíquico; os registros de adoecimento diante de tais eventos são conhecidos na história da psiquiatria (Briere \& Scott, 2015). O Manual Diagnóstico e Estatístico de Transtornos Mentais (DSM) os define como eventos traumáticos: morte, risco de morte ou ameaça à integridade física de pessoas. A definição é base para a classificação de quadros 
nosológicos ligados ao trauma e ao estresse, com destaque para o Transtorno de Estresse Pós-Traumático - TEPT (APA, 2013).

Mas seriam também um fator de promoção da saúde? A ideia de que é possível identificar mudanças positivas na vida das pessoas após a vivência de um evento traumático não é nova, está presente na filosofia, nos ensinamentos religiosos e ditos populares (Zoellner \& Maercker, 2006). De fato, alguns autores afirmam que crescimento pessoal paralelo ao sofrimento é possível. A hipótese foi denominada Crescimento Pós-Traumático - CPT (Tedeschi \& Calhoun, 2004).

O modelo de Richard Tedeschi e Lawrence Calhoun (Tedeschi \& Calhoun, 1996) tem se destacado no estudo sobre o CPT. Trata-se de uma formulação teórica que caracteriza o construto como tridimensional, a saber: 1) percepção de mudanças em si mesmo $($ self $), 2)$ percepção de mudanças nas relações com os demais e 3) percepção de mudanças na filosofia de vida.

Em contextos ocupacionais, o estudo do CPT é especialmente relevante no setor de emergências, dada a natureza das tarefas desenvolvidas. Policiais, bombeiros e profissionais de ambulância vivenciam eventos traumáticos de forma contínua. Suas vidas são colocadas em risco quando combatem o crime, extinguem incêndios ou resgatam vítimas de acidentes automobilísticos (Violanti, 2014; Meyer et al., 2012). É também reconhecida a exposição indireta ou vicária: os trabalhadores têm contato próximo com pessoas traumatizadas e sofrem ao escutarem os relatos ou testemunharem as tragédias vividas por outros (Cohen \& Collens, 2013). Ademais, convivem com eventos de magnitude variada, desde aqueles considerados ordinários em serviços de emergências (acidentes automobilísticos, incêndios de pequena proporção, atendimento a vítimas em geral), até os extraordinários (desastres, catástrofes, guerras, atentados terroristas). A exposição ocupacional a eventos traumáticos é denominada "estressores operacionais" (van der Velden, Kleber, Grievink, \& Yzermans, 2010).

A forma como o trabalho é organizado nos serviços de emergências também merece destaque. Diante de situações extremas em que há morte ou risco de morte, os profissionais diferem das vítimas por estarem inseridos em organizações de trabalho (Lima \& Assunção, 2011). Em consequência, suas ações são limitadas por regras ou protocolos de atendimento, dependem do apoio social de chefes e colegas ou podem variar em intensidade e ritmo a depender das diretrizes de contratação de pessoal (Violanti, 2014). Tais condições, quando precárias, são denominadas estressores organizacionais (van der Velden et al., 2010).
Não obstante, as justificativas elencadas, o interesse no CPT no setor de emergências ainda é recente. Cohen e Collens (2013), em uma revisão de estudos qualitativos, sugerem que estresse e crescimento não são excludentes. Ademais, mudanças positivas no funcionamento psíquico parecem ser gradativas e contínuas entre os trabalhadores. A revisão trouxe avanços para a área, mas ainda restam lacunas. Dentre elas, destacam-se a ausência da estimativa de prevalência e de fatores individuais e do contexto associados ao CPT.

Inquéritos de saúde (quantitativos) podem oferecer informações que não estão disponíveis em estudos qualitativos. Qual a prevalência de CPT em profissionais de emergências? Tipos diferentes de eventos traumáti$\cos$ (estressores operacionais) interferem no CPT (por exemplo: eventos ordinários $v s$. extraordinários; diretos $v s$. indiretos)? Estressores organizacionais (demanda de trabalho, controle sobre as tarefas, apoio social, reconhecimento e tipo de vínculo de emprego) têm influência sobre o desfecho? A presente revisão busca responder a estas perguntas. $\mathrm{O}$ objetivo foi realizar uma revisão sistemática da literatura no intuito de estimar a prevalência de CPT entre profissionais de emergências. A revisão buscou ainda identificar estressores ocupacionais (operacionais e organizacionais) associados ao desfecho.

\section{Método}

\section{Registro e Protocolo}

Os autores iniciaram a revisão sobre CPT em profissionais de emergências em 01 de fevereiro de 2017. A revisão foi baseada no método PRISMA (Moher et al, 2009). O estudo foi submetido à International prospective register of systematic reviews (PROSPERO) e aprovado em 08 de novembro de 2017 (número CRD 42017077848). A estratégia vai ao encontro do método PRISMA e visa evitar vieses de decisão post hoc, como a publicação seletiva de resultados. O registro incluiu informações sobre objetivos da revisão, estratégia de busca de material, população de interesse, desfecho, variáveis explicativas e ações para analisar e evitar vieses. O protocolo completo está disponível on-line.

\section{Critérios de Elegibilidade}

Cinco critérios de elegibilidade dos estudos a serem incluídos nesta revisão foram utilizados e são descritos a seguir: a) Tipo de estudo: estudos observacionais de natureza quantitativa. Quanto ao delineamento, foram aceitos estudos caso-controle, coorte (prospectivo ou retrospectivo) e transversais; 
b) Idioma e ano de publicação: artigos publicados em inglês, português ou espanhol, independentemente do ano de publicação; c) Tipo de participantes: trabalhadores ativos que atuam em serviços de emergências; exemplos: bombeiros, policiais, profissionais do setor saúde (médicos, enfermeiros, auxiliares de enfermagem) e pessoal de ambulância (socorristas ou paramédicos). Foram excluídos estudos com indivíduos cuja atuação em situações de emergências não implicam em vínculo de trabalho (estudantes); d) Tipo de exposição: exposição direta ou indireta a eventos traumáticos, eventos traumáticos ordinários (acidentes automobilísticos, incêndios de pequena proporção, atendimento a vítimas em geral), eventos traumáticos extraordinários ou extremos (desastres, catástrofes, guerras), exposição a estressores organizacionais (demanda de trabalho, controle sobre as tarefas, apoio social, recompensa no trabalho e tipo de vínculo de emprego) (Nieuwenhuijsen, Bruinvels, \& Frings-Dresen, 2010); e) Tipo de desfecho: Crescimento Pós-Traumático (CPT) avaliado por meio de instrumentos validados (escalas ou entrevistas).

Foram excluídos construtos correlatos cuja natureza não são consequência da exposição a eventos traumáticos. A Resiliência, por exemplo, diz respeito à capacidade do indivíduo continuar em frente, manter-se firme e saudável em momentos difíceis, mas não é uma consequência da exposição a eventos. Otimismo e Hardiness, também apontados como construtos correlatos, são traços de personalidade o que os diferenciam do conceito de CPT defendido por Tedeschi e Calhoun (2004). Há ainda diferenças marcantes em relação ao Coping, construto que se refere aos esforços cognitivos e comportamentais utilizados para lidar com uma situação estressora. A literatura mostra que esse construto é um mediador da relação entre trauma e CPT. O debate sobre uma possível sobreposição de construtos delimita ainda diferenças entre CPT e Autoeficácia e Senso de Coerência (Tedeschi \& Calhoun, 2004).

\section{Fontes de Informação: Bases de Busca}

A busca foi realizada nas seguintes bases de dados: Medical Literature Analysis and Retrieval System Online (Medline - via PubMed), Literatura Latino-Americana e do Caribe em Ciências da Saúde (Lilacs - via BVS) e Scientific Electronic Library Online (SciELO).

\section{Estratégias de Busca}

As estratégias para cada base foram baseadas na combinação de dois grupos de descritores; o primeiro consistiu em termos relativos aos trabalhadores em foco; o segundo consistiu no termo "crescimento pós-traumático". Ambos foram combinados por meio de operadores booleanos (OR para combinações intragrupo; AND para aquelas entre grupos).

Grupo 01: Emergency Responders, Emergencies, Emergency Nursing, Emergency Service Hospital, Emergency Medical Technicians, Emergency Medical Services, Emergency Police Dispatcher, Emergency Medical Dispatcher, Emergency Medical Dispatch, Ambulances, Air Ambulances, Nurses, Nurses' Aides, Fires, Firefighters, Police.

Grupo 02: Posttraumatic Growth.

Os descritores foram utilizados em português, inglês e espanhol. A busca nas bases de dados foi complementada pela análise das referências citadas nos artigos encontrados.

\section{Fluxo de Seleção de Artigos e Análise dos Dados}

A seleção das referências foi conduzida em duas etapas: 1) leitura dos títulos e resumos; e 2) leitura dos artigos na íntegra. Ambas as etapas foram realizadas por avaliadores independentes (EPL, AGV). Incongruências foram discutidas com um terceiro avaliador (EN).

Para a extração de dados, o primeiro autor (EPL) desenvolveu um formulário prévio, submetido à aprovação dos demais autores. O formulário previu a coleta dos seguintes dados: país em que o estudo foi realizado, ano de coleta de dados, ano de publicação do estudo, tipo de instrumento de coleta de dados (para desfecho e estressores ocupacionais), categoria profissional estudada, informações sobre população e amostra do estudo, objetivos do estudo, critérios de inclusão e exclusão dos participantes, variáveis estudadas (tipo de exposição ocupacional), tipo de análise estatística (descritiva, uni e multivariada), resultados relativos ao desfecho, e resultados relativos à associação entre estressores ocupacionais e desfecho. A extração foi realizada de forma independente por dois avaliadores (EPL, AGV) e discutida com um terceiro em caso de discordância (EN). O formulário de extração completo e formatado pode ser obtido com os autores do presente estudo.

Os dados extraídos foram organizados em uma planilha no Excel $^{\circledR}$. Os artigos foram analisados quanto ao país de origem, categoria profissional, informações sobre população e amostra (incluindo cálculo amostral), informações sobre instrumentos de coleta para avaliação da exposição a estressores operacionais e organizacionais e CPT (incluindo informações sobre 
validação dos instrumentos), média e desvio padrão dos participantes no instrumento de mensuração do CPT e resultados da associação entre estressores ocupacionais e CPT.

\section{Análise da Qualidade dos Estudos e Risco de Viés}

Para avaliar a qualidade metodológica dos estudos e o risco de viés, foram considerados os critérios propostos por Durant (1994) e as recomendações de Sanderson, Tatt e Higgins (2007) para estudos observacionais. Cada artigo inclú́do na revisão foi analisado quanto a treze características (formuladas como perguntas): $\mathrm{C} 1$ - Os objetivos do estudo foram claramente apresentados? C2 - Os procedimentos metodológicos selecionados foram apropriados para testar as hipóteses do estudo? C3 - Os critérios de inclusão e exclusão dos participantes foram apresentados? C4 - Informações sobre a população do estudo foram claramente apresentadas? C5 - Informações sobre a amostra (e o processo de amostragem) foram claramente apresentadas? C6 - A amostra selecionada foi adequada para testar as hipóteses do estudo? C7 - As medidas de avaliação do desfecho foram claramente apresentadas?; C8 - As medidas de avaliação do desfecho foram validadas? C9 - As medidas de avaliação das variáveis explicativas foram claramente apresentadas? C10 - As medidas de avaliação das variáveis explicativas foram validadas? C11 - Os procedimentos de análise dos dados foram claramente apresentados? C12 - Os procedimentos de análise dos dados foram apropriados para testar as hipóteses do estudo? C13 - Os procedimentos de análise de dados consideraram possíveis fatores de confusão?

A análise para cada critério foi realizada por dois avaliadores independentes (EPL, AGV) e discutida com um terceiro avaliador em caso de divergência (EN). Para cada item foi atribuído as seguintes classificações: sim, não, parcialmente e dados faltantes (nas situações em que o artigo não apresentava informação suficiente para tomada de decisão).

\section{Análise da Associação entre Escores no ICPT e Fatores Ocupacionais}

Para a identificação dos estressores ocupacionais associados, foram analisados os valores de correlação bivariada, regressão logística ou razão de chances (Odds Ratio - OR) e regressão linear, sendo consideradas significativas apenas aquelas cujo p-valor foi menor ou igual a $0,05 \mathrm{em}$ todas as análises apresentadas (uni e multivariadas).

\section{Resultados e Discussão}

\section{Informações Gerais sobre o Processo de Revisão Sistemática}

O fluxo completo do processo de identificação, seleção e inclusão de artigos é apresentado na Figura 1. A busca inicial nas bases de dados resultou em 58 publicações (mais quatro publicações localizadas nas referências de artigos analisados durante a revisão). Após a retirada de artigos duplicados $(n=3)$, a leitura dos títulos e resumos resultou na exclusão de 36 estudos em função das seguintes características: não abordavam amostra de trabalhadores ativos $(n=60)$, foram publicados em outros idiomas que não aqueles listados nos critérios de inclusão $(n=2)$, utilizaram abordagem qualitativa $(n=5)$ ou revisão teórica $(n=1)$, e não focalizaram amostras de profissionais de emergências $(n=$ 28). Ao final dessa etapa (leitura de títulos e resumos), foram selecionados 23 estudos.

A etapa seguinte consistiu na leitura completa dos artigos. Foram excluídos aqueles que não analisaram o CPT como uma variável dependente $(n=6)$, não utilizaram instrumento validado para avaliar o CPT $(n=1)$ e não apresentaram resultados específicos por categoria de profissionais de emergências $(n=1)$. Ao final, foram inseridos 15 estudos no corpus da revisão.

\section{Informações Gerais sobre os Estudos Analisados}

Os estudos incluídos foram conduzidos em países da Ásia (Palestina e Israel), América do Norte (EUA), Europa (Alemanha, Espanha, Holanda, Itália, Polônia, Reino Unido, República Tcheca) e Oceania (Austrália), publicados entre 2003 e 2017. Quanto às categorias ocupacionais, foram incluídos bombeiros $(n$ $=4)$, enfermeiros $(n=5)$, médicos $(n=2)$, policiais $(n$ $=4)$ e socorristas ou profissionais de ambulância $(n=$ 2). Ao todo, 5.128 trabalhadores foram avaliados nos inquéritos revisados.

Quanto ao instrumento de mensuração do CPT, todos utilizaram o Inventário de Crescimento Pós-Traumático (ICPT) (Tedeschi \& Calhoun, 1996), em versão completa $(n=13)$ ou reduzida $(n=2)$. Vale notar ainda que o foco das investigações abrangeu tanto o CPT clássico ou decorrente da exposição direta a traumas no trabalho quanto o CPT vicário ou resultante do contato próximo com as vítimas. Todos os estudos foram transversais (Tabela 1).

\section{Análise da Qualidade e Risco de Vieses}

A análise da qualidade e risco de vieses dos estudos mostrou que informações sobre objetivos e 


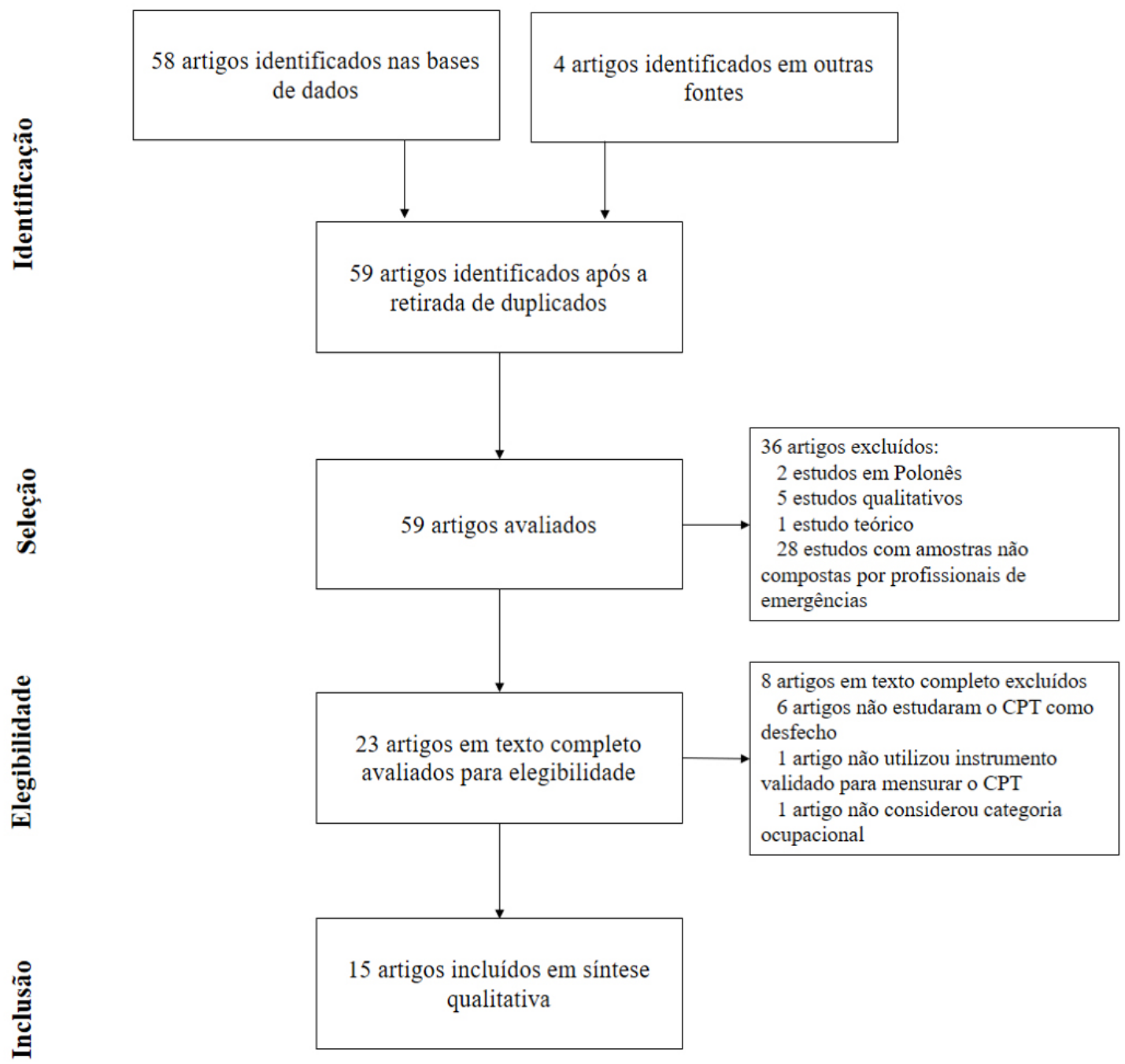

Figura 1. Fluxograma da revisão sistemática sobre CPT e estressores ocupacionais em profissionais de emergências.

procedimentos adotados para atingi-los foram adequados em todos os artigos analisados. Informações sobre o instrumento de mensuração do CPT também foram satisfatórias em 15/15 estudos; todos utilizaram instrumentos validados (ICPT ou ICPT versão resumida). Situação menos favorável foi observada na mensuração da exposição a estressores ocupacionais: $8 / 15$ estudos utilizaram instrumentos validados para tais variáveis.

O principal problema metodológico diz respeito à amostra dos estudos: apenas 3/15 apresentaram informações satisfatórias. Informações sobre a população de referência, cálculo do tamanho amostral, aleatorização, estratégias de recrutamento e taxa de resposta foram omitidas na maior parte das publicações. As informações completas sobre a qualidade e risco de vieses estão dispostas na Tabela 2 .

\section{Prevalência de CPT}

Os escores no ICPT foram analisados a partir da média dos itens (escore total dividido pelo número de itens da escala). A escolha foi justificada pela variação no escore total para as formas completa (0 a 105) e resumida (0 a 50) do ICPT. As médias variaram entre $1,32(d p=1,11)$ em bombeiros que atuavam em diferentes países da União Europeia (Kehl, Knuth, Hulse, \& Schmidt, 2015) e 3,17 ( $d p=0,71)$ em enfermeiros de Israel (Taubman-Ben-Ari \& Weintroub, 2008). Não foi possível identificar a prevalência do CPT nas amostras estudadas, uma vez que o ICPT foi analisado apenas como variável contínua.

Associação entre Estressores Ocupacionais e CPT

A avaliação de estressores ocupacionais (operacionais e organizacionais) compôs o objetivo de todos os 


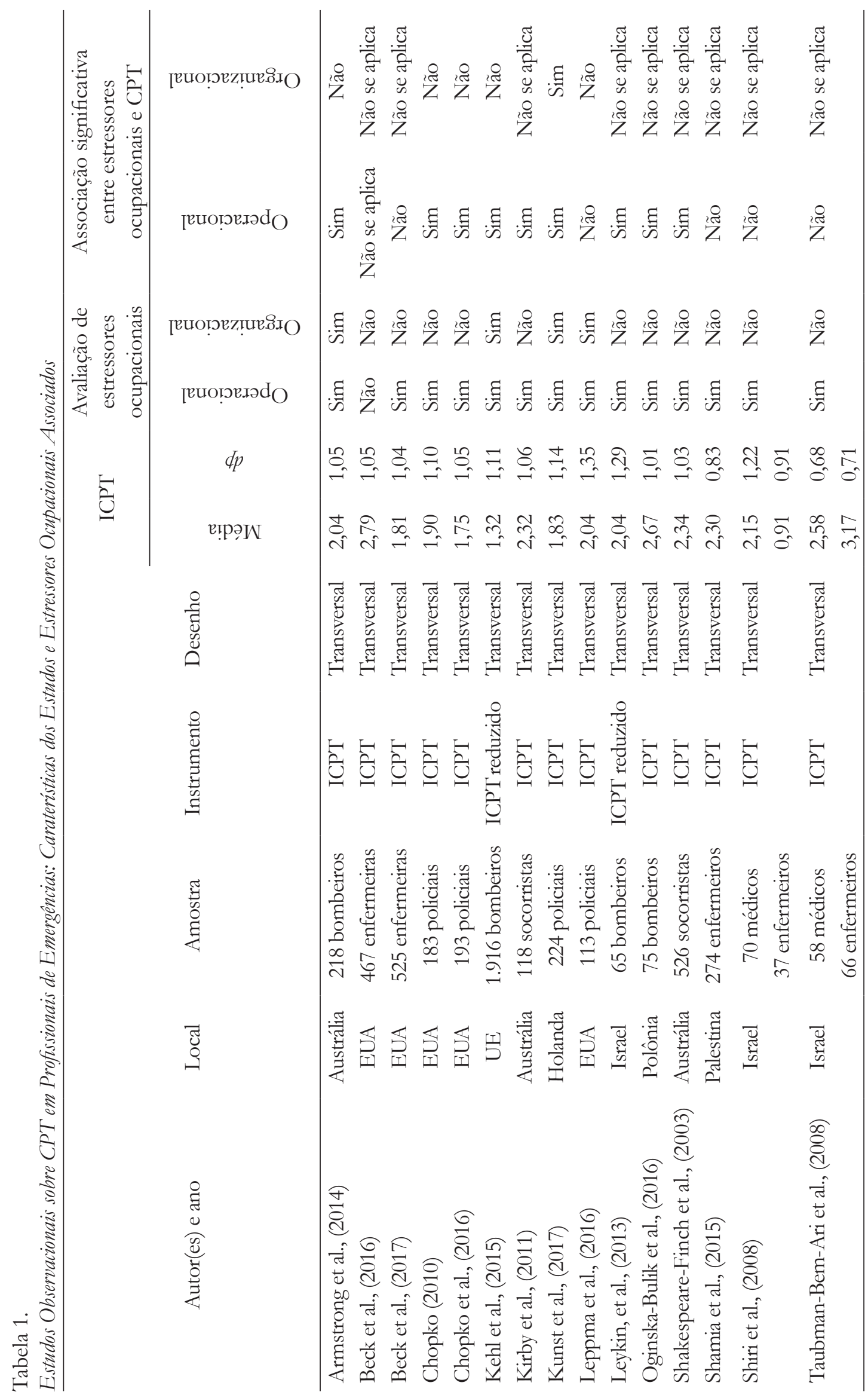


Tabela 2.

Avaliação da Qualidade e Risco de Viés dos Estudos Incluidos na Revisão Sistemática

\begin{tabular}{|c|c|c|c|c|c|c|c|c|c|c|c|c|c|}
\hline Autor(es) / ano & C1 & $\mathrm{C} 2$ & C3 & $\mathrm{C} 4$ & C5 & C6 & $\mathrm{C} 7$ & C8 & C9 & $\begin{array}{c}C \\
10\end{array}$ & $\begin{array}{c}C \\
11\end{array}$ & $\begin{array}{c}\mathrm{C} \\
12\end{array}$ & $\begin{array}{c}C \\
13\end{array}$ \\
\hline Armstrong et al., (2014) & $S$ & S & S & $\mathrm{N}$ & $\mathrm{N}$ & $?$ & $S$ & S & S & $\mathrm{PA}$ & $\mathrm{S}$ & $\mathrm{S}$ & S \\
\hline Beck et al., (2016) & S & S & S & $\mathrm{N}$ & PA & $\mathrm{N}$ & S & S & S & $\mathrm{S}$ & $\mathrm{N}$ & S & $?$ \\
\hline Beck et al., (2017) & S & S & $\mathrm{S}$ & $\mathrm{N}$ & $\mathrm{N}$ & $\mathrm{N}$ & S & S & S & $\mathrm{N}$ & S & S & $?$ \\
\hline Chopko (2010) & S & S & $\mathrm{N}$ & $\mathrm{N}$ & $\mathrm{N}$ & $?$ & S & S & S & $\mathrm{N}$ & S & S & S \\
\hline Chopko et al., (2016) & S & S & $\mathrm{N}$ & $\mathrm{N}$ & $\mathrm{N}$ & $?$ & S & S & S & S & S & S & S \\
\hline Kehl et al., (2015) & S & S & $\mathrm{S}$ & $\mathrm{N}$ & $\mathrm{N}$ & $?$ & S & S & S & S & S & S & S \\
\hline Kirby et al., (2011) & S & S & S & $\mathrm{N}$ & $\mathrm{N}$ & $?$ & S & S & PA & $\mathrm{N}$ & S & S & S \\
\hline Kunst et al., (2017) & S & S & S & S & S & $\mathrm{S}$ & S & S & S & S & S & S & S \\
\hline Leppma et al., (2016) & S & S & S & S & S & $\mathrm{N}$ & S & S & S & PA & S & S & S \\
\hline Leykin, et al., (2013) & S & S & $\mathrm{N}$ & S & S & S & S & S & S & S & S & S & $\mathrm{N}$ \\
\hline Oginska-Bulik (2016) & S & S & S & $\mathrm{N}$ & $\mathrm{N}$ & $\mathrm{N}$ & S & S & S & S & S & S & S \\
\hline $\begin{array}{l}\text { Shakespeare-Finch et al., } \\
(2003)\end{array}$ & S & S & S & $\mathrm{N}$ & $\mathrm{N}$ & $?$ & S & S & $\mathrm{N}$ & $?$ & $\mathrm{~N}$ & PA & $\mathrm{N}$ \\
\hline Shamia et al., (2015) & S & S & S & S & PA & $?$ & S & S & S & S & S & S & $\mathrm{N}$ \\
\hline Shiri et al., (2008) & S & S & S & $\mathrm{N}$ & $\mathrm{N}$ & $?$ & S & S & S & $\mathrm{N}$ & S & S & S \\
\hline $\begin{array}{l}\text { Taubman-Bem-Ari et al., } \\
\text { (2008) }\end{array}$ & S & S & S & $\mathrm{N}$ & $\mathrm{N}$ & $?$ & S & S & $\mathrm{S}$ & PA & S & S & S \\
\hline
\end{tabular}

Nota. C1 a C11: critérios para avaliar a qualidade metodológica dos estudos e o risco de viés; C1 - Objetivos foram apresentados? C2 - Procedimentos metodológicos foram apropriados? C3 - Critérios de inclusão e exclusão dos participantes foram apresentados? C4 - Informações sobre a população do estudo foram apresentadas? C5 - Informações sobre a amostra foram apresentadas? C6 - A amostra selecionada foi adequada? C7 - As medidas de avaliação do desfecho foram apresentadas? C8 - Medidas de avaliação do desfecho foram validadas? C9 - Medidas de avaliação de variáveis explicativas ocupacionais foram apresentadas? C10 - Medidas de avaliação de variáveis explicativas ocupacionais foram validadas? C11 - Procedimentos de análise dos dados foram apresentados? C12 - Procedimentos de análise dos dados foram apropriados? C13 - Procedimentos de análise de dados consideraram fatores de confusão? S - Sim; N - Não; PA - Parcialmente; ? - indica que os dados foram insuficientes para avaliação.

estudos analisados, com exceção de um inquérito sobre CPT em enfermeiras estadunidenses (Beck, Eaton, \& Gable, 2016). Estressores operacionais foram mais frequentemente estudados: 14/15 artigos analisados abordaram o tema. Estressores organizacionais foram considerados em um número menor de publicações (4/15) (Tabela 1).

\section{Estressores Operacionais}

Observa-se que houve associação significativa entre a exposição a estressores operacionais e os escores de CPT em profissionais de emergências em 9/15 artigos nas análises uni e multivariadas (Armstrong, Shakespeare-Finch, \& Shochet, 2014; Chopko, 2010; Chopko, Palmieri, \& Adams, 2016; Kehl et al., 2015; Kirby, Shakespeare-Finch, \& Palk, 2011; Kunst, Saan, Bollen, \& Kuijpers, 2017; Leykin, Lahad, \& Bonneh,
2013; Oginska-Bulik \& Kobylarczyk, 2016; Shakespeare-Finch, Smith, Gow, Embelton, \& Baird, 2003). Entretanto, a classificação do tipo de evento traumático só foi levada em conta em 6/15 estudos.

Quanto à magnitude dos eventos (ordinários vs. extraordinários), apenas um estudo especificou tal parâmetro. Policiais de um departamento do Estado de New Orleans, Estados Unidos, que atuaram durante o Furacão Katrina, foram objeto de um inquérito transversal. O objetivo foi investigar a relação entre o nível de envolvimento (forte, moderado ou leve) no desastre e CPT (Leppma et al., 2017).

Em relação à forma de exposição (direta $v$ s. indireta), apenas 5/15 artigos fizeram tal classificação. Enfermeiras parteiras certificadas pela American College of Nurses-Midwives (ACNM) que prestaram atendimento durante partos traumáticos foram avaliadas por meio 
de instrumentos de autorrelato. A exposição (indireta) foi operacionalizada como número de partos realizados e tempo de duração desde o último parto; sem associações significativas com o desfecho (Beck, Rivera, \& Gable, 2017).

Chopko (2010) avaliou a exposição direta (ser ferido acidentalmente, ser ferido durante agressão, envolver-se em tiroteio) e indireta (testemunhar cenas de morte ou lesões, recolher ou manipular cadáveres, atuar em situações com reféns ou com crianças feridas) a eventos traumáticos entre policiais estadunidenses. Tais situações foram analisadas separadamente em modelos uni e multivariados de regressão linear. Os resultados mostraram que tanto os eventos diretos quanto os indiretos aumentaram as chances de CPT.

Shamia, Thabet e Vostanis (2015) focalizaram a relação entre CPT e eventos traumáticos entre enfermeiros que trabalhavam em áreas de conflito na Faixa de Gaza, Palestina. O estudo foi conduzido após ataques aéreos e terrestres que resultaram em centenas de mortos e milhares de feridos. Os autores mostraram, por meio de um instrumento padronizado - Gaza Traumatic Events Checklist (GTEC), que profissionais expostos a eventos indiretos (testemunhar ataques por tanques de guerra e artilharia pesada e testemunhar a morte de um amigo) apresentaram maiores escores no ICPT.

Duas investigações conduzidas em Israel focalizaram apenas eventos traumáticos indiretos entre médicos e enfermeiros. Na primeira, os participantes foram questionados sobre o número de vítimas de violência atendidas no serviço e tempo transcorrido desde o último atendimento de tal natureza. Os resultados não mostraram associações significativas (Shiri, Wexler, Alkalay, Meiner, \& Kreitler, 2008). A segunda investigação foi apresentada por Taubman-Ben-Ari e Weintroub (2008), que examinaram se a exposição rotineira a morte (número de crianças que faleceram durante tratamento nos últimos seis meses) aumentou o senso de CPT. A hipótese não foi confirmada.

Conclui-se que há associação entre eventos traumáticos no trabalho e CPT. Entretanto, há inconsistências quanto a quais características dos eventos traumáticos influenciam o desfecho. A maior parte das investigações que especificaram a magnitude dos eventos (ordinários ou extraordinários) ou o tipo de exposição (direta ou indireta) não encontrou resultados significativos. É provável que a ausência de instrumentos mais robustos tenha contribuído para o quadro geral apresentado. Vale lembrar: o uso de medidas válidas e precisas tem consequências diretas para a validade dos estudos (Keyes \& Galea, 2014).

\section{Estressores Organizacionais}

Demanda de trabalho, controle sobre as tarefas e reconhecimento não foram objeto de investigação nos artigos incluídos na presente revisão. $\mathrm{O}$ apoio social foi foco de 3/15 estudos. Todos utilizaram instrumentos padronizados para avaliar esse aspecto. Tal característica favoreceu a validade dos resultados. $\mathrm{O}$ primeiro, realizado na Holanda, utilizou os itens sobre apoio social do Job Content Questionnaire (JCQ). No modelo multivariado final, apoio social de chefes e colegas não influenciou o CPT na amostra de policiais em foco (Kunst et al., 2017).

Um segundo estudo com ênfase em apoio social foi apresentado por Leppma et al., (2016). A Interpersonal Support Evaluation List (ISLEL) foi utilizada para avaliar se apoio real (tangível), senso de pertencimento, autoestima ou apoio percebido influenciaram o CPT. Não houve associação entre a exposição e o desfecho.

O terceiro foi focado em bombeiros australianos que vivenciaram pelo menos um evento traumático (Armstrong et al., 2014). Para a avaliação do apoio social, os autores utilizaram o 2-Way Social Support Scale, além de um instrumento geral para estressores ocupacionais: o Operational and Organizational Police Stress Questionnaires (PSQ-Op e PSQ-Org), modificado para bombeiros. A exposição aos estressores em foco não foi associada ao CPT.

A hipótese de associação entre CPT e baixo apoio não foi corroborada; entretanto, sugere-se cautela na interpretação desse resultado. $\mathrm{O}$ apoio social tem se mostrado um bom preditor de transtornos mentais entre trabalhadores (Harvey et al., 2017; Stansfeld \& Candy, 2006). O apoio de chefes e colegas no setor de emergências compreende aspectos emocionais, instrumentais e informacionais; auxiliam na construção de estratégias de enfrentamento e no manejo do estresse no trabalho (Lima \& Assunção, 2011). Seria também um bom preditor de desfechos positivos, como o CP'T? Entende-se que os estudos revisados apresentam limitações para uma resposta segura. Investigações com amostras representativas e com controle adequado de tempo de exposição ocupacional são necessários.

O tipo de vínculo de emprego (profissionais vs. voluntários) foi analisado em um único inquérito (Kehl et al., 2015). Os autores investigaram se bombeiros profissionais diferiam de voluntários em relação aos escores no ICPT. Os resultados indicaram que não 
houve diferença entre os grupos. Entretanto, vale notar que emprego precário é normalmente operacionalizado como vínculo instável ou temporário (Harvey et al., 2017). O CPT e a estabilidade no emprego entre profissionais de emergências estão associados? Os estudos revisados não permitem responder tal questão.

De modo geral, percebeu-se que a avaliação da exposição de profissionais de emergências a estressores organizacionais foi pouco frequente e restrita ao apoio social e ao vínculo de emprego. Pode-se concluir que esse tipo de estressor não é o foco das investigações. Faltam estudos sobre os efeitos de elementos da organização do trabalho cujo papel na explicação da saúde é destacado na literatura, por exemplo, demanda, controle e reconhecimento (Nieuwenhuijsen et al., 2010). A omissão desses elementos como variáveis explicativas nas investigações sobre CPT é uma limitação para o avanço da área, dado o reconhecimento do papel do trabalho sobre a saúde mental da população ativa no mercado de trabalho (Harvey et al., 2017).

Em suma, os resultados da presente revisão indicaram que profissionais de emergências percebem CPT em decorrência da vivência de eventos traumáticos no trabalho, embora não tenha sido possível estudar a prevalência do desfecho. Considera-se que algumas limitações do estudo precisam ser sublinhadas. Em primeiro lugar, foram identificadas falhas na descrição da população e da amostra nos estudos analisados. Tal limitação pode estar ligada a problemas no processo de amostragem, por exemplo ausência de aleatorização e cálculo inadequado de poder estatístico. Ademais, estudos com baixas taxas de resposta ou compostos por amostras de conveniência podem incluir vieses de seleção, ameaçando a validade interna dos inquéritos (Keyes \& Galea, 2014).

Uma segunda limitação é o uso exclusivo do ICPT baseado no modelo de Tedeschi e Calhoun (1996). Zoellner e Maercker (2006), por exemplo, reconhecem a possibilidade de crescimento pessoal após a exposição de eventos traumáticos, mas propõem outra definição: o CPT seria composto por um elemento real (mudanças objetivas no comportamento e nas relações interpessoais) e por um elemento ilusório (mudanças apenas na autopercepção) entre os indivíduos expostos a eventos traumáticos. A proposta de uma dupla face para o CPT foi denominada Modelo Face de Jano. Os autores criticam ainda o uso exclusivo de instrumentos de autorrelato e afirmam que o que se sabe hoje sobre o fenômeno deveria ser definido como CPT autopercebido (que pode diferir do CPT real ou objetivo). A conclusão é que as pesquisas na área precisam investir em medidas objetivas de mensuração do construto.

Uma última limitação a ser destacada é a ausência de estudos longitudinais na presente revisão. Nesse sentido, não é possível excluir a causalidade reversa nas relações entre exposição ocupacional e CPT. Profissionais de emergências mais dispostos a rever suas relações com si mesmos, com os outros e com o mundo seriam mais suscetíveis aos eventos traumáticos no trabalho? Tal hipótese reversa não pode ser descartada apenas com base nos resultados de estudos transversais.

O desenvolvimento de habilidades pode favorecer o crescimento pessoal de profissionais de emergências diante da exposição constante a eventos traumáticos ao longo da carreira (Cohen \& Collens, 2013). Tal perspectiva é coerente com a proposta apresentada nos modelos teóricos sobre CPT (Tedeschi \& Calhoun, 1996; 2004; Zoellner \& Maercker, 2006). Investigações futuras podem contribuir para a estruturação de intervenções organizacionais nessa direção. Finaliza-se, portanto, propondo algumas ações voltadas para a pesquisa sobre CP'T no setor de emergências.

A primeira sugestão é o estabelecimento de um ponto de corte para o ICP'T. A estratégia foi adotada na versão polonesa da escala (Oginska-Bulik \& Kobylarczyk, 2016) e poderá permitir a identificação de níveis de CPT (baixo, médio, alto) experimentado por indivíduos expostos a eventos traumáticos. A análise de prevalência contribuirá para estudos comparativos no setor. Ademais, estabelecer parâmetros mínimos para que os escores no ICPT sejam considerados clinicamente relevantes será útil em estudos de intervenção visando saúde mental no trabalho.

Uma segunda sugestão refere-se ao desenvolvimento de estudos longitudinais, necessários para entender as relações entre exposição ocupacional, TEPT e CPT. Adoecimento e crescimento parecem não ser desfechos excludentes (Armstrong et al., 2014); na verdade, ambos são influenciados pela vivência de eventos traumáticos e parecem resultar de rupturas nas crenças centrais dos indivíduos expostos (Tedeschi \& Calhoun, 2004; Zoellner \& Maercker, 2006). O controle do tempo de exposição e outros fatores de confusão também serão úteis para se conhecer melhor o fenômeno.

Quanto aos estressores ocupacionais, vale enfatizar a importância do uso de instrumentos padronizados. Dentre eles, medidas que não sejam restritas ao autorrelato podem contribuir para o avanço da área (Harvey et al., 2017). Instrumentos válidos e precisos 
podem ser úteis para a estruturação de serviços de saúde adequados às necessidades dos profissionais de emergências, sujeitos a condições de trabalho específicas (Lima \& Assunção, 2011). Vale lembrar que a saúde mental no trabalho não se esgota em questões clínicas, mas também abrange o tipo e a forma de inserção dos trabalhadores no mercado de trabalho (Virtanen et al., 2005).

\section{Considerações Finais}

O CPT é um dos focos em estudos sobre reações pós-traumáticas entre profissionais de emergências. Trata-se de um desfecho positivo, ligado ao desenvolvimento pessoal e profissional. É também o reconhecimento da capacidade de enfrentamento saudável dos trabalhadores diante das adversidades e dos riscos ocupacionais. Estressores operacionais (natureza das tarefas) foram objeto de investigação em grande parte dos estudos e os resultados corroboram a hipótese de associação entre exposição e desfecho. Estressores organizacionais (organização do trabalho) foram raramente investigados, restritos ao apoio social e ao vínculo de emprego. Os resultados para esse segundo grupo de estressores foram inconclusivos.

Diante disso, percebe-se que o tema merece atenção de pesquisadores e profissionais de saúde mental. A produção na área apresenta limitações quanto à qualidade e ao desenho dos estudos. O peso de estressores ocupacionais ainda é subestimado, ora omitido nas investigações, ora mensurado por meio de instrumentos que carecem de padronização e validação. As recomendações apresentadas visam avançar o entendimento sobre o CPT no setor de emergências.

\section{Referências}

American Psychiatric Association. (2013). Diagnostic and Statistical Manual of Mental Disorders - 5th ed. (DSM5). Washington, DC.

Armstrong, D., Shakespeare-Finch, J., \& Shochet, I. (2014). Predicting post-traumatic growth and post-traumatic stress in firefighters. Australian Journal of Psychology, 66(1), 38-46. doi: 10.1111/ajpy.12032

Beck, C. T., Rivera, J., \& Gable, R. K. (2017). A mixed-methods study of vicarious posttraumatic growth in certified nurse-midwives. Journal of Midwifery \& Women's Health, 62(1), 80-87. doi: 10.1111/ jmwh.12523
Beck, C. T., Eaton, C. M., \& Gable, R. K. (2016). Vicarious posttraumatic growth in labor and delivery nurses. Journal of Obstetric, Gynecologic and Neonatal Nursing, 45(6), 801-812. doi: 10.1016/j. jogn.2016.07.008

Briere, J. N., \& Scott, C. (2015). Principles of Trauma Therapy - A Guide to symptoms, evaluation, and treatment (1st. ed). Los Angeles, US: Sage Publication.

Chopko, B. A. (2010). Posttraumatic distress and growth: An empirical study of police officers. American Journal of Psychotherapy, 64(1), 55-72. Recuperado de http://www.ajp.org/archives.html

Chopko, B. A., Palmieri, P. A., \& Adams, R. E. (2016). Posttraumatic growth in relation to the frequency and severity of traumatic experiences among police officers in small to midsize departments. Journal of Interpersonal Violence, pii: 0886260516651089. [ahead of print]. doi: 10.1177/0886260516651089

Cohen, K., \& Collens, P. (2013). The impact of trauma work on trauma workers: A metasynthesis on vicarious trauma and vicarious posttraumatic growth. Psychological Trauma: Theory, Research, Practice, and Policy, 5(6), 570-580. doi: 10.1037/a0030388

Durant, R. H. (1994). Checklist for the evaluation of research articles. Journal of Adolescent Health, 15(1), 4-8. Recuperado de http://www.jahonline.org/ article/1054-139X(94)90381-6/pdf

Harvey, S. B., Modini, M., Joyce, S., Miligan-Saville, J. S., Tan, L., Mykletun, A., Bryant, R.A., Christensen, H., \& Mitchell, P. B. (2017). Can work make you mentally ill? A systematic meta-review of work-related risk factors for common mental health problems. Occupational and Environmental Medicine, 74(4), 301-310. doi: 10.1136/oemed-2016-104015

Kehl, D., Knuth, D., Hulse, L., \& Schmidt, S. (2015). Predictors of postevent distress and growth among firefighters after work-related emergencies - a cross-national study. Psychological Trauma: Theory, Research, Practice, and Policy, 7(3), 203-211. doi: $10.1037 /$ a0037954

Keyes, K. M., \& Galea, S. (2014). Epidemiology matters A new introduction of methodological foundations. New York: Oxford University Press.

Kirby, R., Shakespeare-Finch, J., \& Palk, G. (2011). Adaptative and maladaptative coping Psico-USF, Bragança Paulista, v. 25, n. 3, p. 561-572, jul./set. 2020 
strategies predict posttrauma outcomes in ambulance personnel. Traumatology, 17(4), 25-34. doi: $10.1177 / 1534765610395623$

Kunst, M. J., Saan, M. C., Bollen, L. J. A., \& Kuijpers, K. F. (2017). Secondary traumatic stress and secondary posttraumatic growth in a sample of Dutch police family liaison officers. Stress and Health, 33(5), 570-577. doi: 10.1002/smi.2741.

Leykin, D., Lahad, M., \& Bonneh, N. (2013). Posttraumatic symptoms and posttraumatic growth of Israeli firefighters, at one month following the Carmel Fire Disaster. Psycbiatry Journal, 274121. doi: $10.1155 / 2013 / 274121$

Leppma, M., Mnatsakanova, A., Sarkisian, K., Scott, O., Adjeroh, L., Andrew, M. E., Violanti, J. M., \& McCaniles, E. C. (2016). Stressful life events and posttraumatic growth among police officers: A cross-sectional study. Stress and Health. [Ahead of Print], 1-12. doi: 10.1002/smi.2772

Lima E. P., \& Assunção, A. A. (2011). Prevalência e fatores associados ao transtorno de estresse pós-traumático (TEPT) em profissionais de emergência: Uma revisão sistemática da literatura (Posttraumatic Stress Disorder (PTSD) among firefighters from Belo Horizonte City, Brazil: Prevalence and occupational associated Factors). Revista Brasileira de Epidemiologia, 14(2), 217-230. doi: 10.1590/S1415-790X2011000200004

Meyer, E. C., Zimering, R., Daly, E., Knight, J., Kamholz, B. W., \& Gulliver, S. B. (2012). Predictors of posttraumatic stress disorder and other psychological symptoms in trauma-exposed firefighters. Psychological Services, 9(1),1-15. doi: 10.1037/a0026414

Moher, D., Liberati, A., Altman, D. G., \& PRISMA Group (2009). The PRISMA group. preferred reporting items for systematic reviews and metaanalyses: the PRISMA statement. PLOS Medicine, 339, b2535. doi: 10.1371/journal.pmed1000097

Nieuwenhuijsen, K., Bruinvels, D., \& Frings-Dresen, M. (2010). Psychosocial work environment and stress disorders, a systematic review. Occupational Medicine, 60(4), 277-286. doi: 10.1093/occmed/kqq081

Oginska-Bulik, N., \& Kobylarczyk, M. (2016). Association between resiliency and posttraumatic growth in firefighters: The role of stress appraisal. International Journal of Occupational Safety and Ergonomics, 22(1), 40-48. doi: 10.1080/10803548.2015.1109372
Sanderson, S., Tatt, I. D., \& Higgins, P. T. (2007). Tools for assessing quality and susceptibility to bias in observational studies in epidemiology: A systematic review and annotated bibliography. In ternational Journal of Epidemiology, 36(3), 666-676. doi: 10.1093/ije/dym018

Shamia, N. A., Thabet, A. A. M., \& Vostanis, P. (2015). Exposure to war traumatic experiences, post-traumatic stress disorder and post-traumatic growth among nurses in Gaza. Journal of Psychiatric and Mental Health Nursing, 22(10), 749-755. doi: 10.1111/ jpm.12264

Shakespeare-Finch, J. E., Smith, S. G., Gow, K. M., Embelton, G., \& Baird, L. (2003). The prevalence of post-traumatic growth in emergency personnel. Traumatology, 9(1), 58-71. Recuperado de http:// journals.sagepub.com

Shiri, S., Wexler, I. D., Alkalay, Y., Meiner, Z., \& Kreitler, S. (2008). Positive psychological impact of treating victims of politically motivated violence among hospital-based health care providers. Psychotherapy and Psychosomatics, 77(5), 315-318. doi: 10.1159/000142524

Stansfeld, S., \& Candy, B. (2006). Psychosocial work environment and mental health - A meta-analytic review. Scandinavian Journal of Work and Environmental Health, 32(6, special issue), 443-462. Recuperado de www-jstor-org.ez27.periodicos.capes.gov.br/ stable/40967597

Taubman-Ben-Ari, O., \& Weintroub, A. (2008). Meaning in life and personal growth among pediatric physicians and nurses. Death Studies, 32(7), 621645. doi: 10.1080/07481180802215627

Tedeschi, R. G., \& Calhoun, L. G. (1996). The posttraumatic growth inventory: Measuring the positive legacy of trauma. Journal of Traumatic Stress, 9(3), 455-471. Recuperado de http://www.ncbi.nlm. nih.gov/pubmed/8827649

Tedeschi, R. G., \& Calhoun, L.G. (2004). Posttraumatic growth: Conceptual foundations and empirical evidence. Psychology Inquiry, 15(1), 1-18. doi: 10.1037/0003-066X.55.1.99

van der Velden, P. G., Kleber, R. J., Grievink, L., \& Yzermans, J. C. (2010) Confrontations with aggression and mental health problems in police officers: The role of organizational stressors, life-events and 
previous mental health problems. Psychological Trauma (DNLM), 2, 135-144. doi: 10.1037/a0019158

Violanti, J. M. (2014). Dying for the job - police work exposure and health. New York: Charles C. Thomas Publisher LTD.

Virtanen, M., Kiviäki, M., Joensuu, M., Virtanen, P., Elovainio, M., \& Vahtera, J (2005). Temporary employment and health: A review. International Journal of Epidemiology, 34(3), 610-622. doi: 10.1093/ije/dyi024
Zoellner, T., \& Maercker, A. (2006). Posttraumatic growth in clinical psychology - A critical review and introduction of a two component model. Clinical Psychology Review, 26(5), 626-653. doi: 10.1016/j. cpr.2006.01.008

Recebido em: 11/10/2018

Reformulado em: 18/05/2019

Aprovado em: 09/09/2019

Sobre os autores:

Eduardo de Paula Lima é psicólogo (2003), mestre em Psicologia (2005) e doutor em Saúde Pública (2013) pela Universidade Federal de Minas Gerais. Realizou residência doutoral na Université Laval (Quebec, Canadá), em 2012, e residência pós-doutoral na Birkebeck, Univeristy of London (Londres, Inglaterra), em 2018. Atualmente, realiza residência pós-doutoral no Programa de Pós-Graduação em Psicologia da UFMG (2019-atual). É oficial psicólogo no Corpo de Bombeiros Militar de Minas Gerais. Tem interesse em Saúde do Trabalhador e Profissionais de Emergências.

ORCID: https://orcid.org/0000-0001-8957-8025

E-mail:edpl@hotmail.com

Alina Gomide Vasconcelos é psicóloga (2007), mestre em Psicologia (2010), doutora em Neurociências (2012) pela Universidade Federal de Minas Gerais (UFMG), colaboradora da Rede Aferição e oficial psicóloga no Corpo de Bombeiros Militar de Minas Gerais (CBMMG). Realizou residência pós-doutoral no Programa de Pós-Graduação em Psicologia da UFMG (2015-2017) e na Birkbeck, Univeristy of London (Londres, Inglaterra) (2018) com o projeto Estudo da Saúde do Bombeiro.

ORCID: https://orcid.org/0000-0003-2406-1812

E-mail: alinagomide@gmail.com

Elizabeth do Nascimento é psicóloga (1987), mestre em Psicologia (1993) pela Universidade Federal de Minas Gerais, doutora em Psicologia pela Universidade de Brasília (2000) e professora associada da Universidade Federal de Minas Gerais. Tem experiência na área de Psicologia, com ênfase em Construção e Validade de Testes e, recentemente, tem se dedicado a temas em saúde ocupacional.

ORCID: https://orcid.org/0000-0002-3244-2271

E-mail: bethdonascimento@gmail.com

Contato com os autores:

Av. Alfredo Balena, 190/705

Belo Horizonte-MG, Brasil

CEP: 30310-100 